\title{
Entanglement by a beam splitter: Nonclassicality as a prerequisite for entanglement
}

\author{
M. S. Kim, ${ }^{1}$ W. Son, $, 2, *$ V. Bužek, ${ }^{3, \dagger}$ and P. L. Knight ${ }^{4}$ \\ ${ }^{1}$ School of Mathematics and Physics, The Queen's University, Belfast BT7 1NN, United Kingdom \\ ${ }^{2}$ Department of Physics, Sogang University, CPO Box 1142, Seoul, Korea \\ ${ }^{3}$ Institute of Physics, Slovak Academy of Sciences, Dúbravská cesta 9, 84228 Bratislava, Slovakia \\ ${ }^{4}$ Optics Section, Blackett Laboratory, Imperial College, London SW7 2BW, United Kingdom
}

(Received 20 June 2001; published 27 February 2002)

\begin{abstract}
A beam splitter is a simple, readily available device which can act to entangle output optical fields. We show that a necessary condition for the fields at the output of the beam splitter to be entangled is that the pure input states exhibit nonclassical behavior. We generalize this proof for arbitrary (pure or impure) Gaussian input states. Specifically, nonclassicality of the input Gaussian fields is a necessary condition for entanglement of the field modes with the help of a beam splitter. We conjecture that this is a general property of beam splitters: Nonclassicality of the inputs is a necessary condition for entangling fields in a beam splitter.
\end{abstract}

DOI: 10.1103/PhysRevA.65.032323

PACS number(s): 03.67. $-\mathrm{a}, 89.70 .+\mathrm{c}$

\section{INTRODUCTION}

Entanglement is at the heart of the current development of quantum information processing [1]. Entanglement-assisted communication can enlarge the channel capacity [2] and enhance channel efficiency [3]. Entanglement may play a key role in secure communication [4]. In quantum computation, of course, qubits are massively entangled.

The generation and characterization of entanglement have been studied extensively. In particular, a recent experimental advance realized the generation and distillation of polarization-entangled photons toward optimal entanglement in a $(2 \times 2)$-dimensional Hilbert space [5]. The polarizationentangled photons are generated using type I or type II parametric down-conversion. Parametric down-conversion is also a standard technique to produce a two-mode squeezed state, which is an entangled state in an infinite dimensional Hilbert space [6].

The beam splitter is also one of the few experimentally accessible devices that may act as an entangler. There have been some previous studies of a beam splitter as an entangler [7-9]. In particular, Paris [9] studied entanglement properties of the output state from a Mach-Zehnder interferometer for squeezed input states. The action of a linear directional coupler can also be described by the beam splitter operator. Photon statistics and nonclassical properties of the output fields from a linear directional coupler have been studied for Fock and squeezed inputs [10].

In this paper we investigate the entangling properties of a beam splitter for various pure input states including Fock states and squeezed states. We find a simple formula to determine the entanglement of the output fields for squeezed input fields. We also study the entanglement of the output fields when the input fields are in a Gaussian mixed state and provide a sufficient condition for input fields to have no en-

\footnotetext{
*Also at Institute of Quantum Information Processing and Systems, The University of Seoul, Dongdaemun-Gu, Seoul, Korea.

${ }^{\dagger}$ Also at Faculty of Informatics, Masaryk University, Botanická 68a, 60200 Brno, Czech Republic.
}

tanglement in the output state: when two Gaussian "classical" fields are input to the beam splitter, the output state is never entangled. We find that entanglement of the output state is strongly related to the nonclassicality of the input fields.

\section{FOCK-STATE INPUT}

Figure 1 shows the schematic arrangement of a beam splitter. The input field described by the operator $\hat{a}$ is superposed on the other input field with operator $\hat{b}$ by a lossless symmetric beam splitter, with amplitude reflection and transmission coefficients $r$ and $t$. The output-field annihilation operators are given by

$$
\hat{c}=\hat{B} \hat{a} \hat{B}^{\dagger}, \quad \hat{d}=\hat{B} \hat{b} \hat{B}^{\dagger},
$$

where the beam splitter operator is [11]

$$
\hat{B}=\exp \left[\frac{\theta}{2}\left(\hat{a}^{\dagger} \hat{b} e^{i \phi}-\hat{a} \hat{b}^{\dagger} e^{-i \phi}\right)\right]
$$

with the amplitude reflection and transmission coefficients

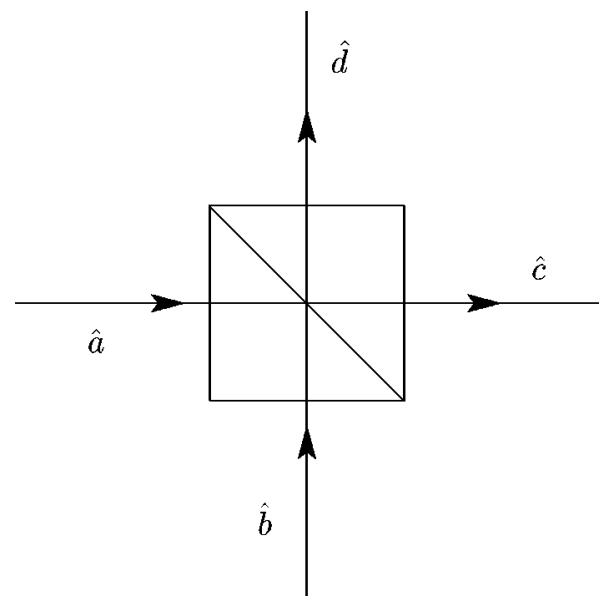

FIG. 1. Configuration of the beam splitter operation. 


$$
t=\cos \frac{\theta}{2}, \quad r=\sin \frac{\theta}{2} .
$$

The beam splitter gives the phase difference $\phi$ between the reflected and transmitted fields.

In this paper we are interested in the entanglement properties of the output state. Suppose that input states are two independent Fock states $\left|n_{1}, n_{2}\right\rangle \equiv\left|n_{1}\right\rangle_{a}\left|n_{2}\right\rangle_{b}$. The output fields are then a superposition of two-mode Fock states:

$$
\begin{aligned}
\hat{B}\left|n_{1}, n_{2}\right\rangle & =\sum_{N_{1} N_{2}}\left|N_{1}, N_{2}\right\rangle\left\langle N_{1}, N_{2}|\hat{B}| n_{1}, n_{2}\right\rangle \\
& =\sum_{N_{1} N_{2}} B_{n_{1} n_{2}}^{N_{1} N_{2}}\left|N_{1}, N_{2}\right\rangle,
\end{aligned}
$$

where

$$
\begin{aligned}
B_{n_{1} n_{2}}^{N_{1} N_{2}}= & e^{-i \phi\left(n_{1}-N_{1}\right)} \sum_{k=0}^{n_{1}} \sum_{l=0}^{n_{2}}(-1)^{n_{1}-k} r^{n_{1}+n_{2}-k-l} t^{k+l} \\
& \times \frac{\sqrt{n_{1} ! n_{2} ! N_{1} ! N_{2} !}}{k !\left(n_{1}-k\right) ! l !\left(n_{2}-l\right) !} \delta_{N_{1}, n_{2}+k-l} \delta_{N_{2}, n_{1}-k+l}
\end{aligned}
$$

with $\delta$ a Kronecker delta function. When the total number of input photons is $N=n_{1}+n_{2}$, the output state becomes an $(N+1)$-dimensional entangled state.

The von Neumann entropy is a measure of entanglement for pure bipartite states (see, e.g., [12]), which becomes $\ln (N+1)$ when an $(N+1)$-dimensional bipartite system is maximally entangled. The von Neumann entropy $E\left(\hat{\rho}_{c}\right)$ for the reduced density operator $\hat{\rho}_{c}=\operatorname{Tr}_{d} \hat{B}\left|n_{1}, n_{2}\right\rangle\left\langle n_{1}, n_{2}\right| \hat{B}^{\dagger}$ is

$$
E\left(\hat{\rho}_{c}\right)=-\sum_{N_{1} N_{2}}\left|B_{n_{1} n_{2}}^{N_{1} N_{2}}\right|^{2} \ln \left|B_{n_{1} n_{2}}^{N_{1} N_{2}}\right|^{2}
$$

Figure 2 shows the von Neumann entropy $E\left(\hat{\rho}_{c}\right)$ as a function of the reflection coefficient $r$ and configuration of input photon numbers. It is interesting to note that the entropy does not necessarily maximize for a 50:50 beam splitter. This is discussed further in the following subsections.

\section{A. $S U(2)$ coherent state}

When $N$ number of photons are injected into one input port while no photon is injected into the other input port, the output state turns into a state generally known as an SU(2) coherent state $[13,14]$. Substituting $n_{1}=0$ and $n_{2}=N$ into Eq. (4), we find the $\mathrm{SU}(2)$ coherent state

$$
\hat{B}|0, N\rangle=\sum_{k=0}^{N} c_{k}^{N}|k, N-k\rangle,
$$

where

$$
c_{k}^{N}=\left(\begin{array}{c}
N \\
k
\end{array}\right)^{1 / 2} r^{k} t^{N-k} e^{i k \phi} .
$$

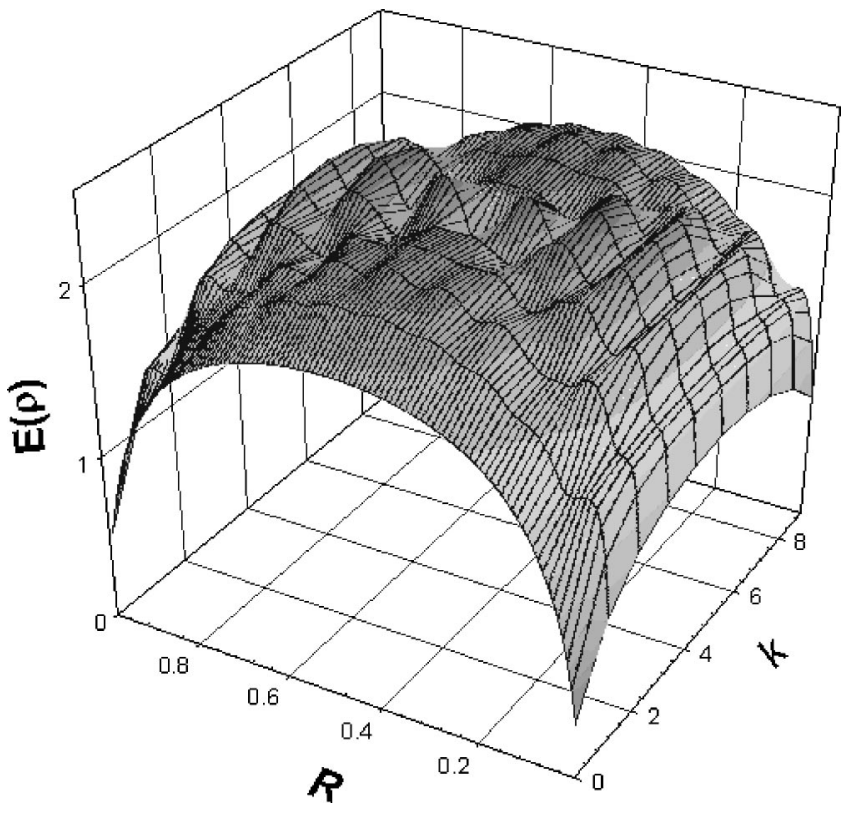

FIG. 2. The measure of entanglement $E(\hat{\rho})$ is plotted using the von Neumann entropy for the reduced density operator of the beamsplitter output field. The Fock-state input fields $|k, N-k\rangle$ have total photon number $N=10 . R \equiv r^{2}$.

The von Neumann entropy for the reduced density operator $\hat{\rho}_{c}$ is $E\left(\hat{\rho}_{c}\right)=\sum_{k=0}^{N}\left|c_{k}^{N}\right|^{2} \ln \left|c_{k}^{N}\right|^{2}$. In Fig. 2, the von Neumann entropy for $N=10$ is plotted, which shows that the measure of entanglement is a convex function with its maximum for a 50:50 beam splitter, i.e., $r=t=1 / \sqrt{2}$. In particular, when $N$ $=1$ the output state is $1 / \sqrt{2}(|0,1\rangle+|1,0\rangle)$ for a $50: 50$ beam splitter $[7,15]$.

\section{B. Input fields of same number of photons}

In Fig. 2, it is interesting to note that, for a 50:50 beam splitter, the entanglement shows a dip when $n_{1}=n_{2}$. When the two input Fock states have the same number of photons, i.e., $n_{1}=n_{2}=n$, the output state is

$$
\begin{aligned}
\hat{B}|n, n\rangle= & \sum_{m=0}^{n} e^{-i(n-2 m) \phi\left(\frac{1}{2}\right)^{n} \sum_{k=0}^{n}(-1)^{n-k}\left(\begin{array}{l}
n \\
k
\end{array}\right)} \\
& \times\left(\begin{array}{c}
n \\
2 m-k
\end{array}\right) \frac{\sqrt{2 m !(2 n-2 m) !}}{n !}|2 m, 2 n-2 m\rangle
\end{aligned}
$$

for a 50:50 beam splitter. This shows that the possibility of having odd numbers of photons is zero [10]. This is an extension of the well-known result of $\hat{B}|1,1\rangle=(1 / \sqrt{2})(|0,2\rangle$ $\left.+e^{i \phi}|2,0\rangle\right)$ [16]. The output state $|1,1\rangle$ may result from transmission or reflection of both the photons. The two cases destructively interfere to remove the $|1,1\rangle$ state in the output state. In fact, the output state is the maximally entangled state in the Hilbert space composed of $|0\rangle$ and $|2\rangle$. We now see why entanglement is not maximized when the same numbers of photons are injected to a 50:50 beam splitter. This is 
due to the fact that odd-number states destructively interfere and do not appear in the output state. It is also true that the output state can be considered in an $(n+1)$-dimensional Hilbert space composed of $|0\rangle,|2\rangle, \cdots,|2 n\rangle$ instead of a $(2 n$ $+1)$-dimensional space.

With the use of a beam splitter, there are two ways to generate entangled states in $(n+1)$-dimensional Hilbert space. One way is to put a total of $n$ photons into a beam splitter and the other way is to put $n$ photons into each input port of a 50:50 beam splitter. By comparing the von Neumann entropies for the two cases, we find that the latter case of using a 50:50 beam splitter does not bring about the best entanglement.

\section{SQUEEZED STATE INPUTS}

Generating Gaussian states, in particular, coherent states and squeezed states has become a standard experimental technique. When two coherent states are incident on a beam splitter, the output is given by

$\hat{B} \hat{D}_{a}(\alpha) \hat{D}_{b}(\beta)|0,0\rangle=\hat{D}_{a}\left(t \alpha+r e^{i \phi} \beta\right) \hat{D}_{b}\left(t \beta-r e^{-i \phi} \alpha\right)|0,0\rangle$

where $\hat{D}(\alpha)=\exp \left(\alpha \hat{a}^{\dagger}-\alpha^{*} \hat{a}\right)$ is the displacement operator [17]. The output state (10) is clearly not entangled. It is further found that displacing the input fields does not increase entanglement of the output fields because the impact of displacing the input fields can always be canceled by local unitary operations on the output fields.

When the two input fields are squeezed, the output state from a beam splitter is

$$
\hat{B} \hat{S}_{a}\left(\zeta_{1}\right) \hat{S}_{b}\left(\zeta_{2}\right)|0,0\rangle
$$

where the squeezing operator [18]

$$
\hat{S}(\zeta)=\exp \left(\frac{1}{2} \zeta * \hat{a}^{2}-\frac{1}{2} \zeta \hat{a}^{\dagger 2}\right)
$$

with the complex squeezing parameter $\zeta=s \exp (i \varphi)$. The phase $\varphi$ of the squeezing parameter determines the direction of squeezing. Using the rotation operator $\hat{R}(\vartheta)$ $=\exp \left(i \vartheta \hat{a}^{\dagger} \hat{a}\right)$ the following can be written:

$$
\begin{aligned}
\hat{B}(\theta, \phi) \hat{S}(\zeta) & =\hat{B}(\theta, \phi) \hat{R}(\varphi / 2) \hat{S}(s) \hat{R}^{\dagger}(\varphi / 2) \\
& =\hat{R}(\varphi / 2) \hat{B}(\theta, \phi-\varphi / 2) \hat{S}(s) \hat{R}^{\dagger}(\varphi / 2),
\end{aligned}
$$

where, in order to specify the parameters $\theta, \phi$ of the beam splitter operator, the beam splitter operator has been denoted by $\hat{B}(\theta, \phi)$. The first rotation operator in the last line of Eq. (13) is canceled by local operation and the last rotation operator does not change the state when it is applied to the vacuum. Now we have found that the relative phase $\phi$ between the amplitude reflection and transmission coefficients gives the effect of the rotation of the squeezing angle for the input fields. Without losing generality, we take the input squeezing parameter to be real while keeping $\phi$ variable.
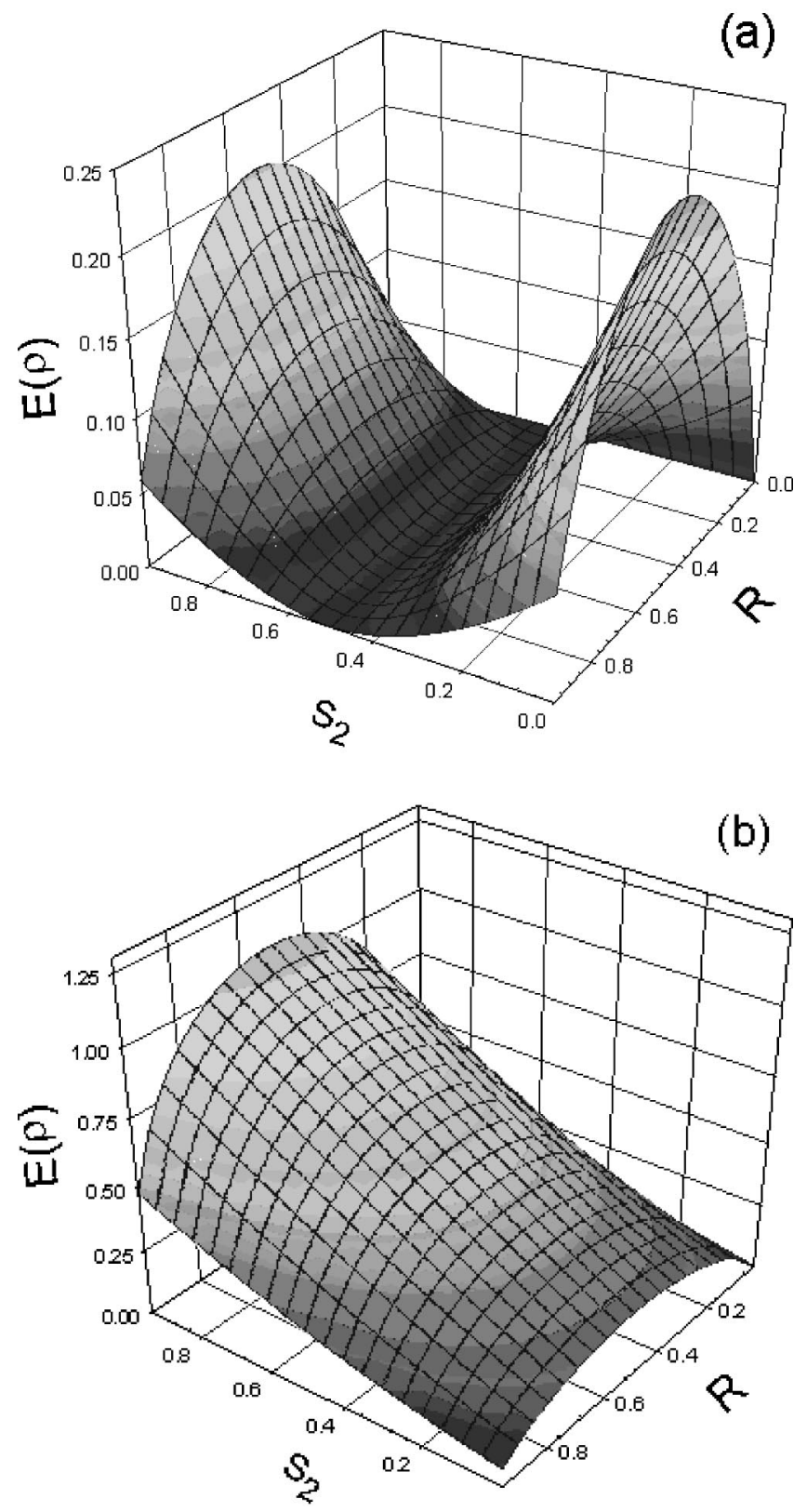

FIG. 3. The measure of entanglement $E(\hat{\rho})$ for the beam-splitter output field is plotted using the von Neumann entropy for the reduced density operator of the output field. The squeezing parameter for one squeezed input is fixed to $s_{1}=0.5$ while the squeezing parameter for the other squeezed state is varied from $s_{2}=0$ to 1 . The transmittivity is $R$. The beam splitter gives phase difference $\phi=0$ (a) and $\phi=\pi / 2$ (b) between the reflected and transmitted fields. $R \equiv r^{2}$.

The von Neumann entropy $E\left(\hat{\rho}_{c}\right)$ of the output state (11) is plotted in Fig. 3 against the squeezing parameter $s_{2}$ and reflection coefficient for $s_{1}=0.5$. The relative phase $\phi=0$ in Fig. 3(a) and $\pi / 2$ in Fig. 3(b). We find that the entanglement of the output state depends on the degrees of squeezing for input fields and the reflection coefficient. We also note that the relative phase $\phi$ and hence the relative angle of squeezing for the input fields play an important role. For a 50:50 beam splitter, the entanglement of the output state is mini- 
mized when $\phi=0$, while it is maximized when $\phi=\pi / 2$. In other words, for $\phi=0$, the entanglement of the output state is maximized if the two input fields are squeezed along the conjugate quadratures in phase space. To analyze the output state (11) further, consider the following relation for a 50:50 beam splitter of $\phi=l \pi / 2 \quad(l=0,1,2, \ldots)$. In this case, the output state (11) can be written as

$$
\begin{aligned}
\hat{B}(\pi / 4, \phi) & \hat{S}_{a}\left(s_{1}\right) \hat{S}_{b}\left(s_{2}\right)|0,0\rangle \\
= & \hat{S}_{a}\left(\frac{1}{2}\left(s_{1}+s_{2} e^{2 i \phi}\right)\right) \hat{S}_{b}\left(\frac{1}{2}\left(s_{1} e^{-2 i \phi}+s_{2}\right)\right) \\
& \times \hat{S}_{a b}\left(\frac{1}{2}\left(s_{1} e^{i \phi}-s_{2} e^{-i \phi}\right)\right)|0,0\rangle,
\end{aligned}
$$

where $\hat{S}_{a b}(\zeta)=\exp \left(-\zeta \hat{a} \hat{b}+\zeta^{*} \hat{a}^{\dagger} \hat{b}^{\dagger}\right)$ is the two-mode squeezing operator. The single-mode squeezing operators $\hat{S}_{a}$ and $\hat{S}_{b}$ on the right-hand side of Eq. (14) do not contribute toward entanglement of the output state because they can be canceled by local unitary operations. Thus only the two-mode squeezing operator $\hat{S}_{a b}$ determines the entanglement of the output state as only it represents a joint action on both pairs of the bipartite system. For a given squeezing $s_{1}$ and $s_{2}$, when $\phi=\pi / 2$, the output state is maximally entangled. When $\phi=0$, entanglement is minimized. In fact, if $s_{1}=s_{2}$ we completely lose entanglement for $\phi=0$. We notice that $a$ two-mode squeezed state is produced from a single-mode squeezed state by the action of a beam splitter and local unitary operations. In contrast to the case of the Fock-state input, the relative phase between reflection and transmission plays an important role for the case of squeezed input fields.

So far, we have studied only pure input states. From what we have learned we can conclude that the nonclassical behavior of the input fields is a necessary condition for the output fields to be entangled. Specifically, the only pure state that does not possess nonclassical properties is a coherent state (its $P$ function is positive well defined; see the discussion in the next section). As is well known coherent inputs never become entangled in the beam splitter, that is, the output can always be written in the factorized form. On the other hand, as we have shown above, nonclassicality of the inputs is not a sufficient condition for entanglement.

\section{GAUSSIAN MIXED STATE INPUT}

When the input fields are mixed, the output fields from a beam splitter are also mixed. A general mixed continuousvariable state is not easy to deal with because of its complicated nature. However, for a Gaussian two-mode state, the separability condition has been studied extensively [19-21].

The separability of a Gaussian state is discussed using quasiprobability functions and their characteristic functions in phase space. There are a group of quasiprobability functions including the Wigner function, the Husimi $Q$ function, and the $P$ function [22]. In particular, the $P$ function can be used as a measure of the nonclassicality of the given field.
For example, if a single-mode state is nonclassical its density operator $\hat{\rho}^{c l}$ can be written as

$$
\hat{\rho}^{c l}=\int P(\alpha)|\alpha\rangle\langle\alpha| d^{2} \alpha,
$$

where the $P$ function $P(\alpha)$ is positive and well behaved.

It has been shown that, if a two-mode Gaussian state is represented by a positive well-behaved $P$ function $P(\alpha, \beta)$, the state is separable $[20,21]$. Suppose two classical states of $P$ functions $P_{a}(\alpha)$ and $P_{b}(\beta)$ are incident on a beam splitter. Using Eq. (15), the density operator for the output state is written as

$$
\begin{aligned}
\hat{B} \int & P_{a}(\alpha) P_{b}(\beta)|\alpha\rangle_{a}\langle\alpha|\otimes| \beta\rangle_{a}\langle\beta| d^{2} \alpha d^{2} \beta \hat{B}^{\dagger} \\
= & \int P_{a}(\alpha) P_{b}(\beta)\left|t \alpha+r e^{i \phi} \beta\right\rangle_{a}\left\langle t \alpha+r e^{i \phi} \beta\right| \\
& \otimes\left|-r e^{-i \phi} \alpha+t \beta\right\rangle_{b}\left\langle-r e^{-i \phi} \alpha+t \beta\right| d^{2} \alpha d^{2} \beta \\
= & \int P_{a}\left(t \gamma-r e^{i \phi} \delta\right) P_{b}\left(r e^{-i \phi} \gamma+t \delta\right)|\gamma\rangle_{a}\langle\gamma| \\
& \otimes|\delta\rangle_{b}\langle\delta| d^{2} \gamma d^{2} \delta .
\end{aligned}
$$

Here $P_{a}\left(t \gamma-r e^{i \phi} \delta\right) P_{b}\left(r e^{-i \phi} \gamma+t \delta\right)$ is the two-mode $P$ function for the output state. Because $P_{a}(\alpha)$ and $P_{b}(\beta)$ are positive well defined under the assumption of classical input fields, $P_{a}\left(t \gamma-r e^{i \phi} \delta\right) P_{b}\left(r e^{-i \phi} \gamma+t \delta\right)$ is also positive well defined. We have proved a sufficient condition for separability of the output state from a beam splitter: when two classical Gaussian input fields are incident on a beam splitter, the output state is always separable. It follows that for creating a Gaussian entangled state with the help of a beam splitter it is necessary that the input exhibits nonclassical behavior.

We have already seen that two nonclassical input fields do not necessarily bring about entanglement in the output state as two squeezed state inputs may not be entangled in the beam splitter. We investigate the entanglement of the output state when two Gaussian mixed states are incident on a beam splitter.

The necessary and sufficient criterion for the separability of a Gaussian mixed state has been studied using the Weyl characteristic function $C^{(w)}(\zeta, \eta)$ [19-21]. For a two-mode Gaussian state of density operator $\hat{\rho}_{a b}$, the Weyl characteristic function [23] $C^{(w)}(\zeta, \eta) \equiv \operatorname{Tr} \hat{\rho}_{a b} \hat{D}_{a}(\zeta) \hat{D}_{b}(\eta)$ can be written as

$$
C^{(w)}(\zeta, \eta)=\exp \left[-\frac{1}{2}\left(\zeta_{i}, \zeta_{r}, \eta_{i}, \eta_{r}\right) M\left(\zeta_{i}, \zeta_{r}, \eta_{i}, \eta_{r}\right)^{T}\right],
$$

where $M$ is a $4 \times 4$ matrix that completely determines the statistical properties of the Gaussian state. Duan et al. [20] found that after some local operations it is possible to transform the state into another that is represented by the matrix 


$$
M^{\prime}=\left(\begin{array}{cccc}
b_{1} & 0 & c_{1} & 0 \\
0 & b_{2} & 0 & c_{2} \\
c_{1} & 0 & d_{1} & 0 \\
0 & c_{2} & 0 & d_{2}
\end{array}\right),
$$

where the parameters $b_{i}, d_{i}$, and $c_{i}$ satisfy

$$
\begin{gathered}
\frac{b_{1}-1}{d_{1}-1}=\frac{b_{2}-1}{d_{2}-1}, \\
\left|c_{1}\right|-\left|c_{2}\right|=\sqrt{\left(b_{1}-1\right)\left(d_{1}-1\right)}-\sqrt{\left(b_{2}-1\right)\left(d_{2}-1\right)} .
\end{gathered}
$$

Note that the parameters $c_{1,2}$ determine the correlation between the two modes. The necessary and sufficient criterion for separability then reads

$$
\left\langle(\Delta \hat{u})^{2}\right\rangle+\left\langle(\Delta \hat{v})^{2}\right\rangle \geqslant q_{o}^{2}+\frac{1}{q_{o}^{2}},
$$

where $q_{o}^{2}=\sqrt{\left(d_{i}-1\right) /\left(b_{i}-1\right)}$ and the two operators $\hat{u}$ and $\hat{v}$ are defined as

$$
\begin{aligned}
& \hat{u}=\frac{q_{o}}{\sqrt{2}}\left(\hat{a}+\hat{a}^{\dagger}\right)-\frac{c_{1}}{\left|c_{1}\right|} \frac{1}{\sqrt{2} q_{o}}\left(\hat{b}^{\dagger}+\hat{b}\right), \\
& \hat{v}=\frac{i q_{o}}{\sqrt{2}}\left(\hat{a}^{\dagger}-\hat{a}\right)-\frac{c_{2}}{\left|c_{2}\right|} \frac{i}{\sqrt{2} q_{o}}\left(\hat{b}^{\dagger}-\hat{b}\right) .
\end{aligned}
$$

When two mixed states of density operators $\hat{\rho}_{a}$ and $\hat{\rho}_{b}$ are input to a beam splitter, the density operator for the twomode output field is $\hat{\rho}_{\text {out }}=\hat{B} \hat{\rho} \hat{B}^{\dagger}$. The Weyl characteristic function for the output field is

$$
C_{o u t}^{(w)}(\zeta, \eta)=C_{a}^{(w)}\left(t \zeta+r e^{i \phi} \eta\right) C_{b}^{(w)}\left(-r e^{-i \phi} \zeta+t \eta\right),
$$

which is obtained using the relation $\hat{B}^{\dagger} \hat{D}_{a}(\zeta) \hat{D}_{b}(\eta) \hat{B}$ $=\hat{D}_{a}\left(t \zeta+r e^{i \phi} \eta\right) \hat{D}_{b}\left(-r e^{-i \phi} \zeta+t \eta\right)$.

\section{A. Squeezed thermal state inputs}

Consider two thermal states of the same average photon number $\bar{n}$. The density operator for the thermal field is [15]

$$
\hat{\rho}_{t h}=\sum_{n} \frac{(\bar{n})^{n}}{(1+\bar{n})^{1+n}}|n\rangle\langle n| .
$$

Suppose the thermal fields are each squeezed before they are mixed at a beam splitter. From the earlier section, we know that two squeezed vacua result in maximum entanglement for the output field when $\phi=\pi / 2$. We thus restrict our discussion to the case $\phi=\pi / 2$ for the study of two squeezed thermal state inputs. We also assume that the incident fields are equally squeezed.

The squeezed thermal field $\hat{S}(s) \hat{\rho}_{t h} \hat{S}^{\dagger}(s)$ is represented by the following characteristic function:

$$
C^{(w)}(\zeta)=\exp \left[-\frac{1}{2}(2 \bar{n}+1) e^{2 s} \zeta_{r}^{2}-\frac{1}{2}(2 \bar{n}+1) e^{-2 s} \zeta_{i}^{2}\right] .
$$

The squeezed thermal state is said to be nonclassical when one of the quadrature variables has its variance smaller than the vacuum limit; the squeezed thermal state of Eq. (25) is nonclassical when [24]

$$
(2 \bar{n}+1) e^{-2 s}-1<0 .
$$

Throughout the paper $s>0$ is assumed without loss of generality.

For the maximum entanglement of the squeezed input, let us consider a 50:50 beam splitter. Substituting $C_{a, b}^{(w)}$ of Eq. (25) into Eq. (23), the matrix elements in Eq. (17) are found:

$$
\begin{aligned}
b_{1}=b_{2}= & d_{1}=d_{2}=\frac{1}{2}(2 \bar{n}+1)\left(e^{2 s}+e^{-2 s}\right), \\
c_{1} & =\frac{1}{2}(2 \bar{n}+1)\left(e^{-2 s}-e^{2 s}\right), \\
c_{2} & =\frac{1}{2}(2 \bar{n}+1)\left(e^{2 s}-e^{-2 s}\right) .
\end{aligned}
$$

The separability condition (21) in this case reads that the output state is separable when $b_{1}-1 \geqslant\left|c_{1}\right|$. Substituting $b_{1}$ and $c_{1}$ in Eq. (27), it is found that the output state is separable when $(2 \bar{n}+1) e^{-2 s}-1 \geqslant 0$. With the help of Eq. (26), we write that the output state is entangled when the squeezed thermal input fields become nonclassical.

\section{B. Squeezed thermal and vacuum input states}

Suppose a squeezed thermal state is incident on one input port and vacuum is incident on the other input port. As was done earlier, we assume $\phi=\pi / 2$ for the beam splitter. In this subsection we release the condition of the 50:50 beam splitter; hence, the output state depends on the reflection coefficient of the beam splitter. The output state is then represented by the matrix $M$ with its elements:

$$
\begin{array}{cc}
b_{1}=r^{2}(2 \bar{n}+1) e^{-2 s}+t^{2}, & b_{2}=r^{2}(2 \bar{n}+1) e^{2 s}+t^{2}, \\
d_{1}=t^{2}(2 \bar{n}+1) e^{-2 s}+r^{2}, & d_{2}=t^{2}(2 \bar{n}+1) e^{2 s}+r^{2}, \\
c_{1}=\operatorname{tr}\left[(2 \bar{n}+1) e^{-2 s}-1\right], & c_{2}=\operatorname{tr}\left[(2 \bar{n}+1) e^{2 s}-1\right] .
\end{array}
$$

The separability criterion (21) takes different forms depending on the positivity of $b_{1}-1$ and $d_{1}-1$ due to the definition of $q_{o}$. When $b_{1}-1 \geqslant 0$ and $d_{1}-1 \geqslant 0$, the separability criterion becomes

$$
\sqrt{\left(b_{1}-1\right)\left(d_{1}-1\right)}+\sqrt{\left(b_{2}-1\right)\left(d_{2}-1\right)} \geqslant\left|c_{1}\right|+\left|c_{2}\right| .
$$

Otherwise the separability criterion is

$$
-\sqrt{\left(b_{1}-1\right)\left(d_{1}-1\right)}+\sqrt{\left(b_{2}-1\right)\left(d_{2}-1\right)} \geqslant\left|c_{1}\right|+\left|c_{2}\right| .
$$


With the use of $b_{1}$ and $d_{1}$ in Eq. (28), we find that both conditions $b_{1}-1 \geqslant 0$ and $d_{1}-1 \geqslant 0$ imply $(2 \bar{n}+1) e^{-2 s}-1$ $\geqslant 0$. In this case, the inequality (29) is always satisfied and the output state is separable. However, when $(2 \bar{n}+1) e^{-2 s}$ $-1<0$, the separability criterion (30) is never satisfied and the output state is entangled. Here, we confirm our earlier finding that the nonclassicality of the input state provides the entanglement criterion for the output state. When a squeezed thermal state and vacuum are incident on a beam splitter, the output state is entangled only if the squeezed thermal state is nonclassical.

\section{Squeezed vacuum and thermal input states}

So far, we found that nonclassicality of the incident field plays an important role in the entanglement of the output field. Let us suppose that one input field is a squeezed vacuum and the other input field is a thermal state. Differently from the earlier cases in this section, one of the input states is always nonclassical while the other is always classical. Substituting the characteristic functions for the thermal state and squeezed state into Eq. (23), the characteristic function for the output field is represented by Eq. (17) with the matrix $M$ in the form Eq. (18), and the matrix elements are

$$
\begin{array}{cl}
b_{1}=(2 \bar{n}+1) r^{2}+e^{-2 s} t^{2}, & b_{2}=(2 \bar{n}+1) r^{2}+e^{2 s} t^{2}, \\
d_{1}=(2 \bar{n}+1) t^{2}+e^{-2 s} r^{2}, & d_{2}=(2 \bar{n}+1) t^{2}+e^{2 s} r^{2}, \\
c_{1}=\operatorname{tr}\left(2 \bar{n}+1-e^{-2 s}\right), & c_{2}=\operatorname{tr}\left(2 \bar{n}+1-e^{2 s}\right) .
\end{array}
$$

These elements do not satisfy conditions (19) and (20). In order to use the separability criterion (21), the output state has to be locally transformed.

Suppose the output fields are squeezed locally. Assuming an equal degree of squeezing $s$ for each mode, the transformed state is represented by $\hat{\rho}_{\text {trans }}=\hat{S}_{a} \hat{S}_{b} \hat{\rho}_{\text {out }} \hat{S}_{a}^{\dagger} \hat{S}_{b}^{\dagger}$. We use the identity $\hat{S}^{\dagger}(s) \hat{D}(\alpha) \hat{S}(s)=\hat{D}\left(\alpha_{r} e^{s}+i \alpha_{i} e^{-s}\right)$, where the subscripts $r$ and $i$, respectively, denote the real and imaginary parts, and definition (17), to find the Weyl characteristic function for the transformed state:

$$
C_{\text {trans }}^{(w)}(\zeta, \eta)=C_{\text {out }}^{(w)}\left(\zeta_{r} e^{s}+i \zeta_{i} e^{-s}, \eta_{r} e^{s}+i \eta_{i} e^{-s}\right),
$$

where $C_{\text {out }}^{(w)}$ is the characteristic function for the output state. After a little algebra, we find that the matrix elements representing $C_{\text {trans }}^{(w)}(\zeta, \eta)$ are the same as those in Eq. (28) for the output state from a beam splitter when the squeezed thermal and vacuum are input fields but with squeezing factor $-s$. The separability criterion $(2 \bar{n}+1) e^{-2 s}-1<0$ thus applies for the output state when the two input fields are the squeezed vacuum and the thermal field. The separability criterion coincides with the nonclassicality condition for the output field of mode $c$ in Fig. 1.

\section{REMARKS}

We have considered the nature of the entanglement of output fields from a beam splitter for pure state inputs and for mixed Gaussian state inputs. In the case of pure states we have found that, for Fock-state inputs, the beam splitter is a tool to produce an $(N+1)$-dimensional entangled state, where $N$ is the total excitation of the input fields. For squeezed vacuum inputs, the entanglement of the output fields depends on many factors including the relative angle of squeezing between the two input fields. When the relative angle is appropriately chosen, the entanglement of the output state is maximized for a 50:50 beam splitter. From these results it directly follows that nonclassicality of the input pure states is a necessary condition for having entangled states at the output of the beam splitter.

In the case of mixed states the analysis is more complicated since there does not exist a necessary and sufficient condition for inseparability of arbitrary infinite-dimensional bi-partite systems. Since the condition exists for Gaussian states, we have concentrated our attention on these states. We have proved a sufficient condition for the output state of a beam splitter to be separable (that is they are not entangled): if both the Gaussian input fields are classical, it is not possible to create entanglement in the output of the beam splitter. From here it automatically follows that nonclassicality is a necessary condition for the entanglement.

These observations make us conjecture that nonclassicality of at least one of the input fields is a necessary condition for the output to be entangled. That is, the nonclassicality of individual inputs can be traded for quantum entanglement of the output of the beam splitter.

Note added in proof. Recently, a paper describing an interesting approach to entanglement generation by passive optical devices including a beam splitter has appeared [25].

\section{ACKNOWLEDGMENTS}

This work was supported by the U.K. Engineering and Physical Sciences Research Council (EPSRC), a BK21 grant of the Korea Ministry of Education, and the European Union project EQUIP under Contract No. IST-1999-11053. W.M. thanks Dr. J. Lee for discussions and the Korean Ministry of Science and Technology through the Creative Research Initiatives program for financial support under Contract No. 00C-CT-01-C-35.
[1] C. H. Bennett and D. P. DiVincenzo, Nature (London) 404, 247 (2000).

[2] C. H. Bennett and S. J. Wiesner, Phys. Rev. Lett. 69, 2881 (1992).

[3] C. H. Bennett, G. Brassard, C. Crepeau, R. Jozsa, A. Peres, and
W. K. Wootters, Phys. Rev. Lett. 70, 1895 (1993).

[4] A. K. Ekert, Phys. Rev. Lett. 67, 661 (1991); W. Tittel, J. Brendel, H. Zbinden, and N. Gisin, ibid. 81, 3563 (1998).

[5] P. G. Kwiat, S. Barraza-Lopez, A. Stefanov, and N. Gisin, Nature (London) 409, 1014 (2001). 
[6] A. Furusawa, J. L. Sørensen, S. L. Braunstein, C. A. Fuchs, H. J. Kimble, and E. S. Polzik, Science 282, 706 (1998).

[7] S. M. Tan, D. F. Walls, and M. J. Collett, Phys. Rev. Lett. 77, 285 (1990).

[8] B. C. Sanders, Phys. Rev. A 45, 6811 (1992); B. C. Sanders, K. S. Lee, and M. S. Kim, ibid. 52, 735 (1995); S. Scheel, L. Knöll, T. Opartiný, and D.-G. Welsch, Phys. Rev. A 62, 043803 (2000)

[9] M. G. A. Paris, Phys. Rev. A 59, 1615 (1999).

[10] W. K. Lai and V. Bužek, Phys. Rev. A 43, 6323 (1991).

[11] R. A. Campos, B. E. A. Saleh, and M. C. Teich, Phys. Rev. A 40, 1371 (1989).

[12] S. J. D. Phoenix and P. L. Knight, Ann. Phys. (N.Y.) 186, 381 (1988).

[13] K. Wodkiewicz and J. H. Eberly, J. Opt. Soc. Am. B 3, 458 (1986)

[14] V. Bužek and T. Quang, J. Opt. Soc. Am. B 6, 2447 (1989).
[15] R. Loudon, The Quantum Theory of Light (Clarendon, Oxford, 2000).

[16] C. K. Hong, Z. Y. Ou, and L. Mandel, Phys. Rev. Lett. 59, 2044 (1987).

[17] R. J. Glauber, Phys. Rev. 131, 2766 (1963).

[18] R. Loudon and P. L. Knight, J. Mod. Opt. 34, 709 (1987).

[19] R. Simon, Phys. Rev. Lett. 84, 2726 (2000).

[20] L.-M. Duan, G. Riedke, J. I. Cirac, and P. Zoller, Phys. Rev. Lett. 84, 2722 (2000).

[21] J. Lee, M. S. Kim, and H. Jeong, Phys. Rev. A 62, 032305 (2000).

[22] K. E. Cahill and R. J. Glauber, Phys. Rev. 177, 1882 (1969).

[23] S. M. Barnett and P. L. Knight, J. Mod. Opt. 34, 841 (1987).

[24] M. S. Kim, F. A. M. de Oliveira, and P. L. Knight, Phys. Rev. A 40, 2494 (1989).

[25] S. Scheel and D.-G. Welsch, Phys. Rev. A 64, 063811 (2001). 